Reprod. Nutr. Dévelop., 1987, 27 (3), 673-679.

\title{
Evolution des dépenses énergétiques chez le rat Zucker au cours de la première semaine de la vie. Effet de l'heure des mesures
}

\author{
Eléonore PLANCHE, M. JOLIFF.
}

Laboratoire sur la Physiopathologie de la Nutrition, INSERM U.177, Institut Biomédical des Cordeliers, 15, rue de l'Ecole de Médecine, 75270 Paris Cedex 06, France.

Summary. Gas exchanges in Zucker rats during the first weeks of life. Effect of measurement time.

Gas exchanges were measured in 2, 5 and 7-day old $\mathrm{fa} / \mathrm{fa}$ and $\mathrm{Fa} / \mathrm{fa}$ rats for two hours (10.30-12.30 h) at five different temperatures : $35,33,30,28$ and $26^{\circ} \mathrm{C}$. In 2-day old pups, no significant difference between genotypes was found at either temperatures. At the age of 5 and 7 days, gas exchanges were significantly lower in $\mathrm{fa} / \mathrm{fa}$ than in $\mathrm{Fa} / \mathrm{fa}$ pups, but only at temperatures below $33^{\circ} \mathrm{C}$. These results differ from those of a previous study where gas exchanges were measured in the afternoon (16.30-18.30 h) (Planche and Joliff, 1985). Thus, our findings show taht very early in life there are diurnal variations in the level of gas exchanges according to genotypes. Consequently, estimation of the deficit of energy expenditure in $\mathrm{fa} / \mathrm{fa}$ rats observed in the present study during the first week of life should be modulated and emphasis laid on the effect of the hour of measurement, even from the early days of life, on the result of metabolic studies.

\section{Introduction.}

Le rôle d'un déficit des dépenses énergétiques, lié à une anomalie de la thermogenèse, dans l'installation et le développement de certaines obésités, notamment d'origine génétique, est maintenant bien établi, grâce en particulier à des études portant sur le rat Zucker, obèse homozygote pour le gène fa, dans les tout premiers jours de la vie (Planche et al., 1983 ; Bazin et al., 1984 ; Schmidt et al., 1984 ; Moore et al., 1985).

Dans un précédent travail nous avons montré que ce déficit énergétique, présent dès l'âge de 2 jours, était largement suffisant pour rendre compte de l'excès de lipides corporels du fa/fa à l'âge de 7 jours (Planche et Joliff, 1985). Dans nos conditions expérimentales (mesures effectuées entre $16 \mathrm{~h} 30$ et $18 \mathrm{~h} 30$ ) les quantités de lipides stockés en excès par le fa/fa, calculées par calorimétrie indirecte et extrapolées aux $24 \mathrm{~h}$, étaient supérieures à celles effectivement mesurées à l'âge de 7 jours (Planche et al., 1983). Ce désaccord pouvait être dû à des variations nycthémérales, dès le début de la vie, dans le niveau des dépenses énergétiques 
et dans les différences entre les génotypes, comme le laissaient supposer les résultats d'une expérience préliminaire portant sur des rats âgés de 7 jours à la température ambiante de $28^{\circ} \mathrm{C}$, au cours de la période diurne (Planche et Joliff, 1985).

Le travail actuel a été entrepris afin d'établir l'âge d'apparition de ces variations et d'apprécier leur impact sur le niveau réel des dépenses énergétiques des ratons Zucker obèses ou non obèses.

\section{Matériel et méthodes.}

Animaux. - Les animaux utilisés sont des rats Zucker obèses (fa/fa) et non obèses ( $\mathrm{Fa} / \mathrm{fa}$ ) âgés de 2,5 et $7 \mathrm{j}$, nés du croisement d'un mâle obèse (fa/fa) et d'une femelle hétérozygote ( $\mathrm{Fa} / \mathrm{fa}$ ). Les petits (8-10 par portée) et leur mère sont conservés dans une animalerie éclairée de 7.00 à $19.00 \mathrm{~h}$ et dont la température est de $22 \pm 1^{\circ} \mathrm{C}$. Les animaux sont étudiés à l'état nourri. Nous avons utilisé 312 animaux : $45 \mathrm{fa} / \mathrm{fa}$ et $51 \mathrm{Fa} / \mathrm{fa}$ à 2 jours, $50 \mathrm{fa} / \mathrm{fa}$ et $56 \mathrm{Fa} / \mathrm{fa}$ à 5 jours, $55 \mathrm{fa} / \mathrm{fa}$ et $55 \mathrm{Fa} / \mathrm{fa}$ à 7 jours. Après l'étude, les petits sont pesés, marqués et rendus à leur mère. Le génotype est identifié à l'âge de 6 semaines.

Echanges gazeux. - Les échanges gazeux sont mesurés dans un appareil à circuit fermé permettant la mesure conjointe de $\mathrm{O}_{2}$ consommé et de $\mathrm{CO}_{2}$ excrété, comme préalablement décrit (Planche et al., 1983). Tous les petits d'une même portée sont étudiés simultanément, chacun d'eux étant placé individuellement dans une chambre métabolique. La quantité $\mathrm{d}^{\prime} \mathrm{O}_{2}$ consommée est mesurée par lecture directe sur un réservoir gradué et corrigée pour la température et la pression (STPD). La quantité de $\mathrm{CO}_{2}$ excrété, collecté dans $8 \mathrm{ml} \mathrm{d}^{\prime} \mathrm{NaOH}$ I $\mathrm{N}$, est mesurée par titrimétrie à l'aide d'un pHstat (Radiometer, Copenhague). Les résultats sont exprimés en $\mathrm{ml} \mathrm{d} \mathrm{O}_{2}$ et de $\mathrm{CO}_{2}$ par $100 \mathrm{~g}$ de poids corporel, pendant $2 \mathrm{~h}$. Les mesures sont effectuées de $10.30 \mathrm{~h}$ à $12.30 \mathrm{~h}$. L'heure de la mesure a été déterminée en fonction des résultats d'une expérience préliminaire (Planche et Joliff, 1985) montrant que, pour la période diurne, le minimum des dépenses et le minimum des différences entre les génotypes, à 7 jours, se situaient à cette période de la journée.

Les animaux sont étudiés à 5 températures ambiantes : $35^{\circ} \mathrm{C}$ (température de neutralité thermique), $33^{\circ}, 30^{\circ}, 28^{\circ}$ et $26^{\circ} \mathrm{C}$ : températures tenant compte de celles de l'environnement habituel des ratons de cet âge (Planche et al., 1983).

Statistiques. - Les résultats sont exprimés en moyenne \pm l'erreur type de la moyenne. Les comparaisons entre les différents groupes d'animaux sont faites en utilisant le test $\mathrm{t}^{\prime}$ de Student.

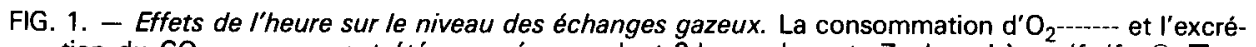
tion du $\mathrm{CO}_{2}$ ont été mesurées pendant $2 \mathrm{~h}$ sur des rats Zucker obèses (fa/fa $\mathrm{O}$ et non obèses $(\overline{\mathrm{Fa} / \mathrm{fa}}) \triangle \Delta$ âgés de 2,5 et 7 jours à 5 températures différentes et à 2 moments de la journée : de $10.30 \mathrm{~h}$ a $12.30 \mathrm{~h}$ (Schéma de gauche) et de $16.30 \mathrm{~h}$ à $18.30 \mathrm{~h}$ (Schéma de droite, reproduit de Planche et Joliff, 1985).

Les différences entre les 2 génotypes sont indiquées par: ${ }^{*} P<0,05 ; * P^{*}<0,01$; *** $P<0,001$. 

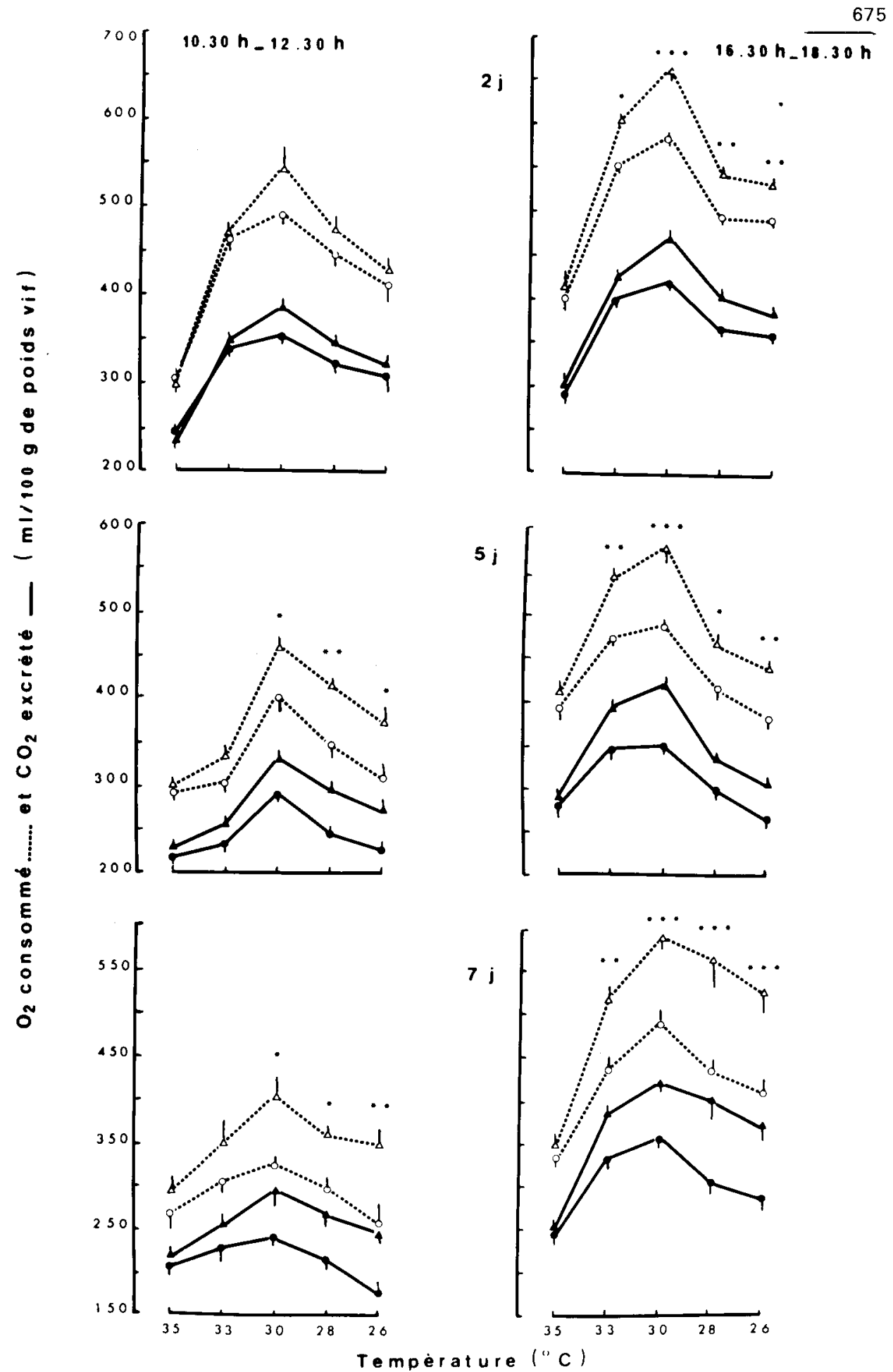


\section{Résultats et discussion.}

Poids des animaux (tabl. 1). - Comme nous l'avons précédemment montré (Planche et al., 1983 ; Planche et Joliff, 1985), aux âges étudiés, le génotype n'a pas d'effet sur le poids corporel des animaux. Ces poids sont tout à fait comparables a ceux des animaux utilisés dans notre précédente étude (Planche et Joliff, 1985).

TABLE 1

Poids des rats $(g)$.

\begin{tabular}{|c|c|c|}
\hline & $\mathrm{fa} / \mathrm{fa}$ & $\mathrm{Fa} / \mathrm{fa}$ \\
\hline 2 jours & $\underset{(45)}{7,23 \pm 0} 0,09$ & $\underset{(51)}{7,24 \pm 0,11}$ \\
\hline 5 jours & $10,31 \pm 0,21$ & $\begin{array}{c}9,94 \pm 0,17 \\
(56)\end{array}$ \\
\hline 7 jours $\ldots \ldots \ldots \ldots \ldots \ldots$ & ${ }_{(55)}^{12,92 \pm 0,20}$ & $\underset{(55)}{12,65 \pm 0,21}$ \\
\hline
\end{tabular}

Nombre de rats entre parenthèses.

Echanges gazeux. - Afin d'illustrer l'effet de l'heure de la mesure sur le niveau des échanges gazeux, nous avons reproduit dans la partie droite de la figure 1 et des colonnes du tableau 2 les résultats des mesures pratiquées de $16.30 \mathrm{~h}$ à $18.30 \mathrm{~h}$ (Planche et Joliff, 1985). Comme nous l'avons précédemment montré (Planche et Joliff, 1983) il n'existait pas, entre les génotypes, de différence dans le niveau du Quotient Respiratoire (Q.R.) qui se situe dans tous les cas entre 0.71 et 0.76 . Ces valeurs peu élevées du Q.R. chez des animaux nourris sont dues probablement à la teneur élevée du lait en lipides (Godbole et York, 1981).

La comparaison des deux séries de détermination montre que, dans les deux génotypes, à tous les âges et pour toutes les températures étudiées, le niveau des échanges gazeux est significativement plus bas (tabl. 2) le matin que le soir. La différence, qui augmente avec l'âge des animaux, est de l'ordre de 10 à $40 \%$ à 2 jours, de 15 à $60 \%$ à 5 jours et de 20 à $70 \%$ à 7 jours, selon la température considérée. Ces résultats corroborent les observations de Schmidt et al. (1984) étudiant les variations de la température centrale de rats Zucker obèses ou non obèses âgés de 2 à $9 \mathrm{j}$. En outre, nos résultats montrent qu'il existe également des variations, liées à l'heure, dans la différence entre les génotypes, les variations dans le niveau des échanges gazeux étant moins accentuées chez les fa/fa que chez les $\mathrm{Fa} / \mathrm{fa}$.

Ainsi, contrairement à ce que nous avions observé en fin d'après-midi, le matin, il n'existe pas de différence significative entre les génotypes chez les animaux âgés de 2 jours à aucune des températures étudiées. Ce fait peut expliquer le désaccord entre nos résultats et ceux de Moore et al. (1985) qui n'observent pas de différence entre obèses et hétérozygotes âgés de 2 jours, leurs mesures, d'après leur protocole, étant pratiquées en fin de matinée. 


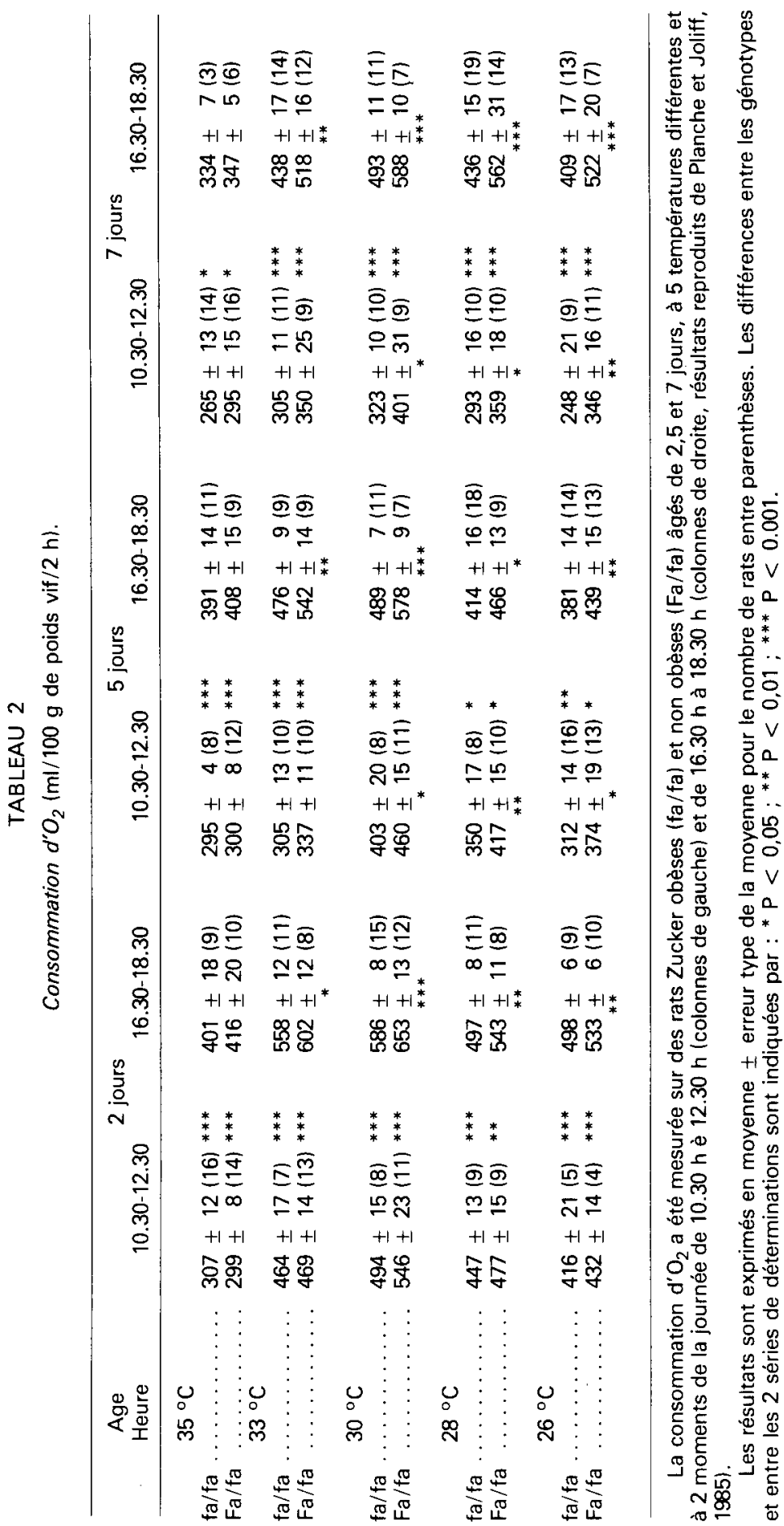


A 5 et 7 jours, la différence entre les génotypes, bien que moins importante que chez les animaux étudiés l'après-midi, est significative à $30^{\circ}, 28^{\circ}$ et $26^{\circ} \mathrm{C}$, mais pas à $33^{\circ} \mathrm{C}$ qui est la température du nid en présence de la mère (Planche et al., 1983). D'après Grota et Ader (1969), pendant la première semaine après la naissance la mère passe 70 à $80 \%$ de son temps sur le nid principalement pendant la période diurne. Il semble donc, à la lumière de ces différentes observations : comportement de la mère et variations dans le niveau des dépenses énergétiques des ratons que, au moins pendant la période diurne, le déficit énergétique du rat $\mathrm{fa} / \mathrm{fa}$ au cours de la première semaine de la vie est moins important que ne le laissait supposer notre précédent travail.

L'existence de rythmes nycthéméraux chez le rat nouveau-né a été peu étudiée. II semble que dans des conditions normales d'environnement, la plupart des rythmes biologiques n'apparaissent que vers la $3^{\text {e }}$ semaine après la naissance. II en est ainsi de la corticostéronémie (Henning, 1978) de la T.R.H. hypothalamique (Martino et al., 1986) ou du niveau des ingesta (Godbole et al., 1981). Par contre, un travail récent de Schmidt et al. (1986) montre l'existence précoce d'un rythme nycthéméral de la température centrale chez des ratons âgés de moins d'une semaine. Nos résultats sont tout à fait en accord avec les observations de ces auteurs et, bien que des mesures ponctuelles à seulement deux moments de la journée ne permettent pas de l'affirmer, ils suggèrent que des variations nycthémérales dans le niveau des dépenses énergétiques, comme dans celui de la température centrale, existent chez le rat nouveau-né au moins dès l'âge de 2 jours.

Ces variations sont probablement induites par la mère, peut-être sous l'influence des variations du taux des hormones dans le lait dont le niveau varie proportionnellement à celui du taux plasmatique maternel (Koldovsky, 1980), et notamment celui des hormones thyroïdiennes (Martin et al., 1978) dont le rôle dans la régulation de la thermogenèse est bien établi (Hogan et Himms-Haggen, 1981 ; Rothwell et al., 1982).

Quoi qu'il en soit, ces variations dans les dépenses énergétiques et surtout dans la différence entre les génotypes, permettent d'expliquer notre surestimation, par le calcul théorique, de l'excès de stockage des lipides chez le $\mathrm{fa} / \mathrm{fa}$ au cours de la première semaine de la vie.

Nos résultats montrent, en outre, chez l'animal nouveau-né comme chez l'adulte, l'importance de l'heure dans l'appréciation de la validité des études métaboliques.

Reçu en août 1986. Accepté en février 1987.

\section{Références}

BAZIN E., ETEVE D., LAVAU M., 1984. Evidence for decreased GDP binding to brown adipose tissue mitochondria of obese Zucker (fa/fa) rats in the very first days of life. Biochem. J., 21, 241-245.

GODBOLE V. Y., GRUNDleger M. L., PASQUine T. A., THENEN S. W., 1981. Composition of rat milk from day 5 to 20 of lactation and milk intake of lean and preobese Zucker pups. J. Nutr., 111, 480-487. 
GROTA L. J., ADER R., 1969. Continuous recording of maternal behaviour in rattus norvegicus. Anim. Behav., 17, 722-729.

HENNING S. J., 1978. Plasma concentrations of total and free corticosterone during development in the rat. Am. J. Physiol., 255 (Endocrinol. Metab. Gastrointest. Physiol. 4), E 451-E 456.

HOGAN S., HIMMS-HAGEN J., 1981. Abnormal brown adipose tissue in genetically obese mice (ob/ob) : effect of thyroxine. Am. J. Physiol., 241 (Endocrinol. Metab. 4), E 436-E 443.

KOLDOWSKY O., 1980. Hormones in milk. Life Sci., 26, 1833-1836.

MARTIN R. J., WANGSNESS P. J., GAHAGAN J. H., 1978. Diurnal changes in serum metabolites and hormones in lean and obese Zucker rats. Horm. Metab. Res., 10, 187-192.

MARTINO E., BAMBINI G., BARTALENA L., AGHINI-LOMBARDI F., BRECCIA M., BASCHIERI L., PINCHERRA A., 1986. Ontegeny of nyctohemeral variations of thyrotropin-releasing hormone in rat hypothalamus. Endocrinology, 119, 232-235.

MOORE B. J., ARMBRUSTER S. J., HORWITZ B. A., STERN J. S., 1985. Energy expenditure is reduced in pre-obese 2-day Zucker fa/fa rats. Am. J. Physiol., 249 (Regulatory Integrative Comp. Physiol. 18), R 262-R 265.

PLANCHE E., JOLIFF M., de GASQUET P., LE LIEPVRE X., 1983. Evidence of a defect in energy expenditure in 7 day old Zucker rat (fa/fa). Am. J. Physiol., 245 (Endocrinol. Metab. 8), E 107-E 113.

PLANCHE E., JOLIFF M., 1985. Evolution des dépenses énergétiques du rat Zucker au cours de la premiere semaine de la vie. Reprod. Nutr. Dévelop., 25, 321-327.

ROTHWELL N. J., SAVILLE M. E., STOCK M. J., WILLIE M. G., 1982. Catecholamines and thyroïd hormone influence on brown fat $\mathrm{Na}^{+}, \mathrm{K}^{+}$AT pase activity and thermogenesis in the rat. Horm. Metab. Res., 14. 261-265.

SCHMIDT I., KAUL R., CARLISTE H. J., 1984. Body temperature of huddling newborn Zucker rats. Pflügers Arch., 401, 418-420.

SCHMIDT I., BARONE A., CARLISLE H. J., 1986. Diurnal cycle of core temperature in hudling, week-old rat pups. Physiol. Behav., 37, 105-109. 